University of Nebraska - Lincoln

DigitalCommons@University of Nebraska - Lincoln

\title{
Adsorbate and thermally induced spin reorientation transition in low-temperature-grown $\mathrm{Fe} / \mathrm{Cu}(001)$
}

\author{
D. Peterka \\ Max Planck Institute for Solid State Research \\ Axel Enders \\ University of Nebraska-Lincoln, a.enders@me.com \\ G. Haas \\ Max Planck Institute for Solid State Research \\ K. Kern \\ Max-Planck-Institut für Festkörperforschung, k.kern@fkf.mpg.de
}

Follow this and additional works at: https://digitalcommons.unl.edu/physicsenders

Part of the Physics Commons

Peterka, D.; Enders, Axel; Haas, G.; and Kern, K., "Adsorbate and thermally induced spin reorientation transition in low-temperature-grown Fe/Cu(001)" (2002). Axel Enders Publications. 5.

https://digitalcommons.unl.edu/physicsenders/5

This Article is brought to you for free and open access by the Research Papers in Physics and Astronomy at DigitalCommons@University of Nebraska - Lincoln. It has been accepted for inclusion in Axel Enders Publications by an authorized administrator of DigitalCommons@University of Nebraska - Lincoln. 


\title{
Adsorbate and thermally induced spin reorientation transition in low-temperature-grown $\mathrm{Fe} / \mathrm{Cu}(001)$
}

\author{
D. Peterka, A. Enders, ${ }^{*}$ G. Haas, and K. Kern \\ Max Planck Institute for Solid State Research, Heisenbergstr. 1, 70569 Stuttgart, Germany
}

(Received 27 March 2002; published 13 September 2002)

\begin{abstract}
Adsorbate and thermally induced changes of the spin reorientation transition in Fe wedges grown at $140 \mathrm{~K}$ on $\mathrm{Cu}(001)$ substrates are investigated by the magneto-optical Kerr effect and Kerr microscopy. The adsorption of oxygen causes a complete reorientation of the easy axis of magnetization into the film plane. In contrast to that carbon monoxide decreases the critical thickness for the reorientation transition of the film by 0.7 monolayer down to 3.6 monolayers, while films thinner than 3.6 monolayers remain magnetized perpendicularly. These adsorbate-induced changes can be ascribed to a change in the surface magnetic anisotropy. Also annealing of the films up to $300 \mathrm{~K}$ causes a temperature-dependent nonlinear shift of the critical thickness. This shift can be separated in reversible and irreversible contributions which arise from temperature-dependent anisotropy constants and thermally induced structural changes of the film, respectively.
\end{abstract}

DOI: 10.1103/PhysRevB.66.104411

PACS number(s): 75.70.Ak, 75.70.Cn

\section{INTRODUCTION}

The structural and magnetic properties of Fe films grown on $\mathrm{Cu}(001)$ have been in the focus of extensive experimental and theoretical work for more than a decade. In the limit of thin films this system shows striking deviations from bulk behavior in structure and magnetism. With increasing film thickness the structural and magnetic properties undergo several transitions. These transitions offer the opportunity to study the correlation between the structure and magnetism of ferromagnetic films. Also, because of the amount of available experimental data, this system is often used as a standard for testing new scientific techniques. Despite considerable effort, open questions concerning the driving force for the magnetic transitions remain.

The focus of the majority of work was on Fe films grown at room temperature (RT). The reason for this is that at 300 $\mathrm{K} \mathrm{Fe}$ films grow in a nearly perfect layer-by-layer mode, which makes it an ideal system for theoretical studies. Recent detailed summaries of the work on this system are given in Refs. 1-4. The literature agrees on the following picture: After nucleation of tetragonally distorted double-layer islands with fct structure a layer-by-layer growth sets in for thicknesses higher than 4 monolayers (ML). Within this $\gamma$-Fe film bcc precipitates can be found with scanning tunneling microscopy (STM). ${ }^{5}$ Above $11 \mathrm{ML}$ the film structure has completely transformed to bcc. Also, with increasing thickness the films go through different magnetic phases. Below $t_{c r i t}=4-5 \mathrm{ML}$ a ferromagnetic phase I with the easy axis perpendicular to the plane is found. This phase is succeeded by an antiferromagnetic phase II, and finally, for $t_{F e}$ $>11 \mathrm{ML}, \quad$ a ferromagnetic phase III with in-plane anisotropy. ${ }^{6}$ Since the thicknesses for magnetic transitions more or less coincide with the structural changes, $\mathrm{fct} \rightarrow \mathrm{fcc}$ $\rightarrow$ bcc, the magnetic phases were ascribed to magnetovolume effects. $^{7,2}$ Theoretical work on the other hand, points out the dominating contribution of the Fe-substrate interface to the magnetic anisotropy energy (MAE) due to electronic effects: namely, band narrowing and hybridization. ${ }^{8}$ Additionally, the antiferromagnetism in phase II is proposed to be due to spin density waves. ${ }^{6,9}$ It has furthermore been shown that the deposition rate has some influence on the interface roughness and the magnetic properties, such as magnetic remanence, coercivity, and live layer Curie temperature. ${ }^{10}$

In this paper we focus on the much less investigated $\mathrm{Fe}$ films grown on $\mathrm{Cu}(001)$ at low temperatures (LT) of $T$ $=140 \mathrm{~K}$. In contrast to RT-grown films, here the easy axis switches from out of plane to in plane directly at a critical thickness of 4.3 ML without exhibiting the intermediate antiferromagnetic phase II. ${ }^{11}$ This reorientation occurs coincidentally with a structural fct $\rightarrow$ bcc transition. Since STM images show a strongly enhanced roughness for LT-grown films, ${ }^{12,13}$ it is assumed that the magnetic properties in this system are governed by reasons other than the electronic structure.

To understand the magnetism in LT-grown films better, we investigated the susceptibility of the spin reorientation transition (SRT) to adsorbate exposure and to thermal treatment. At the critical thickness of the reorientatioin transition the forces favoring perpendicular and in-plane magnetization balance each other. The response of the SRT, i.e., the $t_{c r i t}$, to certain perturbations like adsorbates or temperature therefore helps to reveal its determining factors. As will be shown in this paper, oxygen and carbon monoxide adsorption will considerably shift the SRT to lower thickness. Furthermore, the SRT strongly depends on the annealing temperature within the considered temperature range between $120 \mathrm{~K}$ and RT. The experimental data are explained within the usual picture of a competition between surface anisotropy and magnetostatic energy.

Adsorbates are a powerful tool to investigate magnetism since they can alter the electronic structure of the film in the vicinity of the surface as well as the interlayer spacing. The importance of adsorbates for the surface magnetism was pointed out already, for instance, in early experiments performed on Fe single crystals. ${ }^{14}$ In ultrathin films adsorbateinduced changes can dominate the magnetism of the whole film. This is shown in several experiments with gaseous ${ }^{15,3}$ 
and metallic ${ }^{16}$ adsorbates, as well as for the presence of a gas partial pressure during film growth. ${ }^{2}$

For RT-grown 4- ML Fe films on $\mathrm{Cu}(001)$ hydrogen adsorption induces a reversible transition between the magnetic phases I and II. ${ }^{3} \mathrm{CO}$ adsorption for this system was found to reduce the Curie temperature ${ }^{17}$ the same effect was seen with $\mathrm{Cu}$ cap layers. ${ }^{18}$ Also, small cobalt coverages on LTand RT-grown Fe films are found to cause a spin reorientation into the film plane. ${ }^{16,19}$ Similar to the system $\mathrm{Fe} / \mathrm{Cu}(001)$ investigated in this paper, for $\mathrm{Ni}$ films on $\mathrm{Cu}(001)$ an adsorbate-induced shift of the critical thickness for reorientation was found upon $\mathrm{H}_{2}$ and $\mathrm{CO}$ adsorption and ascribed to a modified surface anisotropy $K_{f-v a c} .{ }^{20}$ The use of oxygen as a surfactant alters the film morphology and the magnetic properties-for instance, in $\mathrm{Co} / \mathrm{Cu}(110){ }^{21}$ These studies clearly show the sensitivity of the film magnetism to electronic modifications in the surface region. The new results in this paper might help to further improve ab initio models of this system. ${ }^{22}$

\section{ANISOTROPY MODEL}

In this section we summarize the relevant energy contributions to the total energy of a thin ferromagnetic film. This model is then used to describe adsorbate and thermally induced changes in the SRT in LT-grown Fe films on $\mathrm{Cu}(001)$.

In general, the total energy of a ferromagnetic thin film can be described as the sum of magnetocrystalline anisotropy $F_{M C}$, the magnetoelastic energy $F_{M E}$, the magnetostatic energy $F_{M S}$, and a surface term $F_{\text {surf }}$ which arises from the broken symmetry and lattice deformations on interfaces, $F_{t o t}=F_{M C}+F_{M E}+F_{M S}+F_{\text {surf }}$. The sign and magnitude of $F_{\text {tot }}$ depend particularly on the direction of magnetization, expressed by the direction cosine $\alpha_{i}$ with respect to the $x, y$, and $z$ directions, and lattice strain with respect to bulk material, $\varepsilon_{i}$. Here, a negative $F_{\text {tot }}$ describes systems with an easy axis perpendicular to the film plane, while a positive $F_{t o t}$ indicates an easy axis within the film plane.

The $F_{M C}$ reflects the symmetry of the crystal lattice and is for a cubic lattice, neglecting terms of higher than fourth order,

$$
F_{M C}=K_{4}\left(\alpha_{1}^{2} \alpha_{2}^{2}+\alpha_{2}^{2} \alpha_{3}^{2}+\alpha_{3}^{2} \alpha_{1}^{2}\right) .
$$

In this definition, a positive value for the fourth- order magnetocrystalline anisotropy constant $K_{4}$ describes a system with the easy axis of magnetization along the [001] directions. The magnetoelastic term describes to what extend lattice strain $\varepsilon_{i}$ contributes to $F_{\text {tot }}$ via the magnetoelastic coupling constants $B_{i}$. The general expression for $F_{M E}$ in cubic systems simplifies to

$$
F_{M E}=B_{1}\left(\varepsilon_{\perp}-\varepsilon_{\|}\right) \alpha_{3}
$$

under the assumption of homogenous in-plane strain $\varepsilon_{\|}$and strain $\varepsilon_{\perp}$ perpendicular to the plane, with respect to bulk lattice dimensions. Expression (2) reflects the difference in magnetoelastic energy between out-of-plane $\left(\alpha_{3}=1\right)$ and inplane $\left(\alpha_{1}=1\right)$ magnetization. If $F_{M E}$ is negative for $\alpha_{3}$
TABLE I. Experimentally obtained magnetic properties of epitaxial Fe films on $\mathrm{Cu}(001)$.

\begin{tabular}{lcc}
\hline \hline Property & Value & Ref. \\
\hline$a_{F e}^{f c c}$ & $3.59 \AA$ & 23 \\
$d(4 \mathrm{ML})$ & $1.87 \AA$ & 24 \\
$\varepsilon_{\|}$ & $1.54 \%$ & \\
$\varepsilon_{\perp}$ & $5 \%$ & \\
$B_{1}^{e f f}\left(\varepsilon_{\|}\right)$ & $13.5 \mathrm{MJ} / \mathrm{m}^{3}$ & 25 \\
$M_{S}$ & $1751 \mathrm{kA} / \mathrm{m}$ & \\
$K_{f-v a c}$ & $0.89 \mathrm{~mJ} / \mathrm{m}^{2}$ & 26 \\
$K_{f-s}$ & $0.6 \mathrm{~mJ} / \mathrm{m}^{2}$ & 27 \\
\hline \hline
\end{tabular}

$\neq 0, F_{\text {tot }}$ is decreased and out-of-plane magnetization is favored. The magnetostatic energy

$$
F_{M S}=\frac{1}{2} \mu_{0} M_{S}^{2} \alpha_{3}^{2}
$$

is a positive energy contribution if the magnetization rotates out of plane, making it energetically unfavorable due to the creation of a magnetic dipole field. Here, the demagnetization factor for thin plates, $D=1$, is already taken into account. Finally, the interface term

$$
F_{\text {surf }}=-\frac{\left(K_{f-v a c}+K_{f-s}\right)}{t_{f}} \alpha_{3}^{2} \approx-\frac{2 K_{S}}{t_{f}} \alpha_{3}^{2}
$$

summarizes all effects which lead to a deviation from bulk behavior and prefer out-of-plane magnetization in the interface anisotropy contributions $K_{f-v a c}$ and $K_{f-s}$, for the surface and film-substrate interface, respectively. The $F_{\text {surf }}$ decays with increasing film thickness $t_{F}$. Both interface anisotropies take into account the modified electronic structure and the different roughness of each interface. Adsorbate-induced changes in the reorientation transition as presented in this paper will be attributed to a change in $K_{f-v a c}$, while in these experiments $K_{f-s}$ remains unchanged. Often though, for simplicity $K_{f-v a c}=K_{f-s}=K_{S}$ is assumed and experimentally confirmed for several systems. To get a criterion for the reorientation transition between out-of-plane and in-plane magnetization, the difference in total energy for a thin film between out-of-plane and in-plane magnetization is calculated using Eqs. (1)-(4), which yields

$$
\Delta F_{t o t}=B_{1}\left(\varepsilon_{\perp}-\varepsilon_{\|}\right)+\frac{1}{2} \mu_{0} M_{S}^{2}-\frac{K_{f-v a c}+K_{f-s}}{t_{F}} .
$$

A change in sign in $\Delta F_{t o t}$ from negative to positive indicates a reorientation transition for the magnetization towards in plane; thus the condition for the critical thickness $t_{\text {crit }}$ results from $\Delta F_{t o t}=0$ :

$$
t_{c r i t}=\frac{2\left(K_{f-v a c}+K_{f-s}\right)}{2 B_{1}\left(\varepsilon_{\perp}-\varepsilon_{\|}\right)+\mu_{0} M_{S}^{2}} .
$$

In Table I all relevant experimental data obtained so far have been summarized. Together with the model given in 
this section they are used in Sec. IV A to draw a complete picture of the anisotropy of low-temperature-grown Fe films on $\mathrm{Cu}(001)$. This model is then used together with the experimental results presented in this paper to deduce adsorbate and thermally induced changes of the surface anisotropy constants.

\section{EXPERIMENTAL PROCEDURE}

The experiments are performed in a UHV chamber with a base pressure of $1 \times 10^{-10}$ mbar. The system combines an experiment to measure the magneto-optical Kerr effect (MOKE), a Kerr microscope, and a magnetic force microscope (MFM). With this unique combination, magnetic domains can be imaged on a scale ranging from $0.4 \mathrm{~mm}$ down to a few $\mathrm{nm}$. External magnetic fields of up to $0.1 \mathrm{~T}$ can be applied parallel and perpendicular to the sample surface. The Kerr microscope is a commercial Jenapol polarization microscope where the sheet polarizers have been replaced by Glan-Thompson prisms. Through a specially designed window a distance between sample surface and objective of 10 $\mathrm{mm}$ is achieved, thus allowing for a magnification of 10 and a resolution of $0.5 \mu \mathrm{m}$. Images are captured with a computer-controlled charge-coupled-device (CCD) camera. Changes in the domain structure or the reorientation transition can thus be observed directly during gas adsorption or thermal treatment, and image sequences can be captured with a repetition rate of 4 s/image.

The sample temperature can be varied from $120 \mathrm{~K}$ by $l N_{2}$ cooling up to $1200^{\circ} \mathrm{C}$ by electron bombardment heating. With a novel tip exchange mechanism ${ }^{28}$ the MFM tip can be replaced by a STM tip and the microscope can thus be operated as an STM. The $\mathrm{Cu}(001)$ substrates are prepared by a standard procedure of three subsequent sputter and annealing cycles. ${ }^{29,30}$ During the last annealing the sample temperature is decreased slowly from $650 \mathrm{~K}$ down to RT with a rate of $\sim 2 \mathrm{~K} / \mathrm{s}$. This procedure has been found the best way to maximize the terrace width, as checked with STM. Fe is evaporated by electron beam heating from a $\mathrm{Fe}$ rod with a purity of $99.99 \%$. During deposition the Fe ion flux is kept constant by adjusting the electron acceleration voltage. The deposition rate is checked with STM with an accuracy better than 0.05 atomic layers.

Several Kerr microscopy experiments have been performed on Fe wedges with a thickness varying from 0 ML up to $12 \mathrm{ML}$. These wedges have been produced by depositing over a straight metal plate mounted on the front end of the $\mathrm{Fe}$ evaporator, thus producing a half shadow region of deposition on the sample surface. In this region of $1.1 \mathrm{~mm}$ in length the film thickness decreases almost linearly from maximum thickness to zero, creating a wedge. The length of the wedge, and thus the slope, was also modified by continuously moving the sample by up to $4 \mathrm{~mm}$ in front of the evaporator during deposition. All results obtained on Fe wedges have been reproduced afterwards on Fe films of constant thickness. Gas adsorption experiments have been performed by adjusting the partial pressure of $\mathrm{H}_{2}, \mathrm{O}_{2}$, or $\mathrm{CO}$ in the UHV chamber between $5 \times 10^{-9}$ and $5 \times 10^{-8}$ mbar. During the

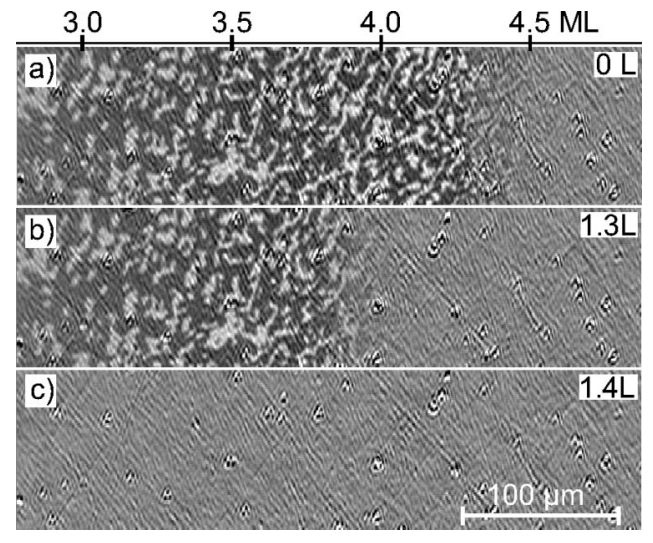

FIG. 1. Kerr images on a Fe wedge grown at $140 \mathrm{~K}$ on $\mathrm{Cu}(001)$ for different oxygen coverages. Adsorption of $1.4 \mathrm{~L} \mathrm{O}_{2}$ leads to a complete reorientation of the magnetization into the plane; thus the magnetic contrast in (c) has disappeared.

experiment the partial pressure has been monitored continuously to determine the gas doses precisely.

\section{RESULTS AND DISCUSSION}

\section{A. Adsorbate-induced-reorientation transition}

We performed several gas adsorption experiments to determine its influence on the magnetic properties of $\mathrm{Fe} /$ $\mathrm{Cu}(001)$. Changes in the reorientation transition of LT-grown Fe films on $\mathrm{Cu}(001)$ due to gas adsorption or heat treatment have been monitored with the Kerr microscope. The advantage of a Kerr microscope to image a large sample region of $400 \mu \mathrm{m} \times 300 \mu \mathrm{m}$ has been exploited to observe changes in the critical film thickness on Fe wedges. Because of the wedge shape of the films, within the field of view of the microscope the film thickness varies by a few monolayer.

The Kerr images in Figs. 1(a)-1(c) show the shift of the critical thickness $t_{c r i t}$ for the SRT between perpendicular and in-plane magnetization due to oxygen adsorption. The asgrown domain state of the Fe wedge near $t_{c r i t}$ is shown in Fig. 1(a). Domains of perpendicular magnetization are visible as a dark-bright contrast in the left part of the image. This domain pattern is somewhat different to the stripe domain pattern of RT-grown Fe wedges as observed by scanning electron microscopy with polarization analysis (SEMPA) in Ref. 31, but similar to domain patterns in RTgrown $\mathrm{Fe}$ as obtained by spin-polarized low energy electron microscopy (SPLEEM). ${ }^{10}$ Above $t_{c r i t}=4.3 \mathrm{ML}$, in the right part of the image, the magnetization lies within the film plane. Our Kerr microscope is much less sensitive to inplane magnetization and therefore no magnetic contrast is visible in this area. One can see clearly in Fig. 1(a) that the SRT does not happen sharply at a certain thickness. Rather, a gradual decay of the magnetic contrast over a thickness range of $0.15 \mathrm{ML}$ is observed, indicating a reorientation via a canted magnetization state.

The adsorption of oxygen strongly alters the magnetic anisotropy in Fe: after exposure to 1.3 Langmuir $(\mathrm{L}) \mathrm{O}_{2}$ the SRT starts to move towards smaller thickness, and after $1.4 \mathrm{~L}$ $\mathrm{O}_{2}$ any perpendicular magnetic contrast has completely dis- 


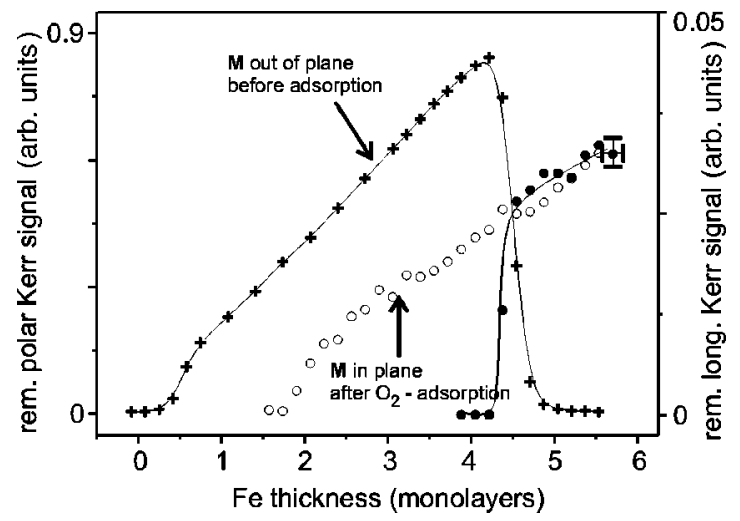

FIG. 2. MOKE on a Fe wedge grown on $\mathrm{Cu}(001)$ at $140 \mathrm{~K}$. The drop of the remanent perpendicular signal prior to adsorption $(+)$ marks the critical thickness of $t_{c r i t}=4.3 \mathrm{ML}$. The remanent inplane Kerr signal after $\mathrm{O}_{2}$ exposure $(O)$ matches the in-plane signal prior to exposure $(\bigcirc)$ for $t_{F e}>4.3 \mathrm{ML}$ within the experimental error.

appeared at all thicknesses [Fig. 1(c)]. Subsequent MOKE experiments on this film reveal the reorientation of the easy axis into the film plane. Figure 2 shows the remanent polar $(+)$ and longitudinal MOKE signal $(\bullet)$ prior to oxygen adsorption as a function of Fe thickness. This dependence of a linear increase of the polar signal with increasing film thickness, followed by a complete drop to zero at $t_{\text {crit }}=4.3 \mathrm{ML}$, is well known for LT-grown $\mathrm{Fe}^{32}$ For films thicker than $t_{\text {crit }}$ only the remanent longitudinal MOKE signal can be measured. After exposing this $\mathrm{Fe}$ wedge to $1.4 \mathrm{~L}$ of $\mathrm{O}_{2}$ the remanent polar MOKE signal completely vanished. Instead, a remanent longitudinal MOKE signal could be measured from a Fe thickness of $2 \mathrm{ML}$ on $(\bigcirc)$. The magnitude of this signal almost precisely overlaps with the longitudinal signal observed prior to oxygen exposure, proving that no magnetic moment of the film is reduced due to the oxygen treatment. The formation of a thorough layer of nonferromagnetic $\mathrm{FeO}$ or $\mathrm{Fe}_{2} \mathrm{O}_{3}$ can therefore be excluded. This experiment clearly shows an oxygen-induced reorientation of the easy axis of magnetization from perpendicular to in-plane in the thickness range between $2 \mathrm{ML}$ and 4.3 ML. Below $2 \mathrm{ML}$ no remanent magnetic signal was detected after $\mathrm{O}_{2}$ exposure. One might speculate whether this rough seed layer mostly consisting of double-layer islands is more susceptible to oxide formation due to the enhanced number of step edge atoms. Also, the Curie temperature of this film might be reduced due to oxygen adsorption.

The adsorbate-induced reorientation is thickness selective when using $\mathrm{CO}$ as an adsorbate. This allows for a more detailed analysis of the change in anisotropy energy by adsorbates. The images in Figs. 3(a) and 3(b) show Kerr images of an $\mathrm{Fe}$ wedge prior and after $\mathrm{CO}$ adsorption, respectively. The critical thickness for the SRT is shifted from 4.3 ML to 3.6 ML by adsorption of $4 \mathrm{~L}$ of CO. In contrast to oxygen adsorption, films thinner than 3.6 ML remain magnetized perpendicularly, even if the film is exposed to $\mathrm{CO}$ doses as high as 20 L [Fig. 3(b)]. This result of a thicknessdependent effect was confirmed on Fe films with constant thickness.

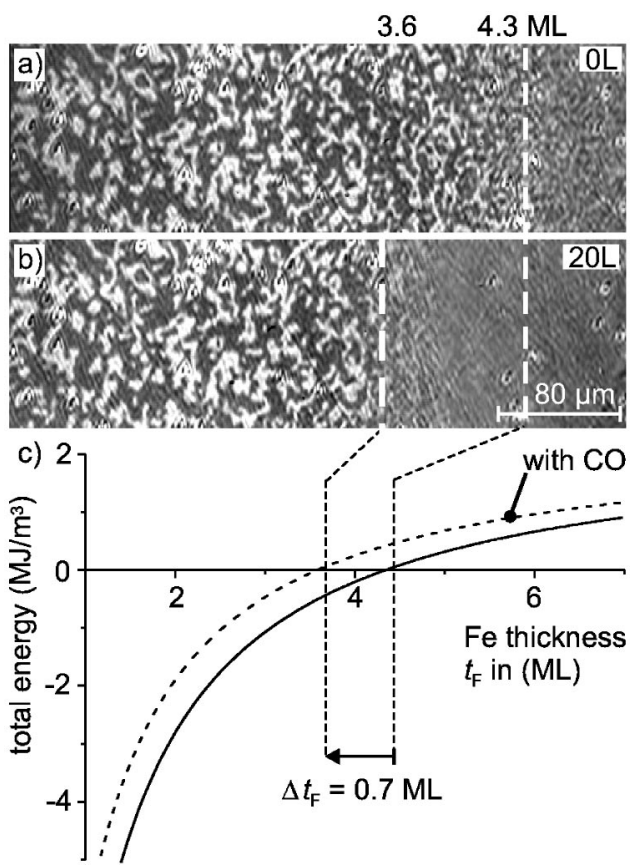

FIG. 3. (a), (b) Kerr image of the SRT before and after CO adsorption. The SRT shifts to $3.6 \mathrm{ML}$ due to CO. No further shift is observed even if $20 \mathrm{~L}$ are applied. (c) The difference of the total energy of the film between perpendicular and in-plane magnetization as a function of thickness (straight line). The change in sign at 4.3 ML marks the critical thickness for reorientation. The COinduced shift can be reproduced with $K_{f-v a c}=0.63 \mathrm{~mJ} / \mathrm{m}^{2}$ (dashed line).

We attribute the reorientation of magnetization to an adsorbate-induced change in the magnetic interface anisotropy energy of the $\mathrm{Fe}$-vacuum interface. The electronic interaction between film and adsorbate is known to alter the surface anisotropy as was investigated for instance for $\mathrm{Ni}$ films on $\mathrm{Cu}(001)$ experimentally ${ }^{20}$ and confirmed by $a b$ initio calculations. ${ }^{33}$ For $\mathrm{O}_{2}$ and $\mathrm{CO}$ adsorbates a strong influence on the electronic structure of Fe films was observed in several experiments. The interaction of $\mathrm{CO}$ with heteroepitaxial fcc and bcc Fe films on $\mathrm{Cu}(001)$ was investigated by ultraviolet photoemission spectroscopy (UPS) and thermal desorption spectroscopy (TDS). ${ }^{34} \mathrm{CO}$ adsorbs on fcc Fe as a molecule in bridge bonding sites, with the $\mathrm{C}$ atom pointing towards the surface. The electron transfer between the $\mathrm{CO}$ molecule and the $\mathrm{Fe}$ is commonly described by the Blyholder model: ${ }^{35}$ Upon chemisorption of $\mathrm{CO}$ the nonbonding $5 \sigma-\mathrm{CO}$ molecular orbital (MO) donates part of its electronic charge into Fe band states, counterbalanced by a backdonation of charge density from the metal to the antibonding $2 \pi^{*} \mathrm{MO}$ of the $\mathrm{CO}$, thus weakening the intramolecular bonding. Due to the particular upright attachment of the $\mathrm{CO}$ to the metal surface, this effect is rather weak compared to the "lyingdown" adsorption geometry on bcc Fe. A much stronger electronic interaction with major consequences for the film magnetism is found for oxygen adsorption on $\mathrm{Fe}^{36}$ The density of states and the spin polarization of Fe near $E_{F}$ decrease gradually with $\mathrm{O}$ coverage. A charge transfer from iron to oxygen leaving behind $\mathrm{Fe}$ states with majority character was 
suggested. On the other hand, for coverages below $1.6 \mathrm{~L}$ the $\mathrm{O}_{2 p}$ states are spin polarized and show ferromagnetic coupling with the substrate. Similar effects were observed for Fe films on different substrates. ${ }^{37}$

Although a conclusive picture about the difference between $\mathrm{O}$ and $\mathrm{CO}$ adsorption cannot be drawn from this comparison, these experimental results indicate a much stronger electronic interaction between $\mathrm{O}$ and the Fe film. In the case of $\mathrm{CO}$ the interaction is reduced because of the particular adsorption geometry of the molecules. We presume that this bears the origin of the difference in the shifts of the critical thickness as shown in this paper, i.e., the complete reorientation of the magnetization due to $\mathrm{O}$ adsorption compared to the rather small effect in the case of CO. For a more detailed understanding of the electronic interaction $a b$ initio calculations are necessary. Spišak and Hafner predicted profound modifications of the electronic and magnetic properties of $\gamma-\mathrm{Fe} / \mathrm{Cu}(001)$ due to chemisorbed $\mathrm{CO}$ by using firstprinciples local spin density calculations. ${ }^{22}$ Spin-orbit coupling was not taken into account in these calculations, and thus an adsorbate-induced reorientation of the magnetization, as observed in our Kerr studies, could not be modeled. Yet a reduced magnetic moment and a change in layer spacing due to magnetovolume effect is predicted. However, a more detailed theoretical model seems desirable and might be stimulated by these experiments.

There is a lot of experimental evidence for electronic interactions between the film and adsorbate as well as for adsorbate-induced changes of the magnetic properties of Fe/ $\mathrm{Cu}(001)$. The usual picture of a balance between the surface and volume anisotropy as the determining factor for the direction of the easy axis of magnetization, as described in Sec. II, can thus be applied. In this picture, this balance is intercepted by the fcc to bcc structural transformation, to which the SRT was attributed originally. ${ }^{32}$ Strong support of this idea comes from experiments using Co capping layers with an opposite effective anisotropy constant to induce a spin reorientation in $\mathrm{Fe}^{16}{ }^{16}$ Also, recent experimental results with $\mathrm{Cu}$ cap layers on this system rather emphasize the dominant role of the outer $\mathrm{Fe}$ interface on the magnetism. ${ }^{18}$ On the other hand, theoretical models by Ujfalussy et al. attribute the perpendicular anisotropy predominantly to electronic effects on the Fe-substrate interface. ${ }^{8}$ This model implies that the film should be less sensitive to manipulation of the outer Fe-vacuum interface.

Hydrogen adsorption on RT-grown Fe films was found to cause a reversible shift of the fct-fcc transition towards higher Fe thickness and an irreversible shift of the fcc-bcc transition. ${ }^{3}$ The former is accompanied by an increase of the coercivity, probably caused by the coexistence of out-ofplane- and in-plane domains. On our LT-grown films, though, we did not see any changes of the domain state or a shift of the SRT due to hydrogen adsorption, even after exposures as high as $50 \mathrm{~L}$.

The shift of $t_{\text {crit }}$ in Fig. 3(b) can be used to estimate the CO-induced change of the surface anisotropy $K_{f-v a c}$, using Eq. (6) and experimental data obtained on this system. For the equilibrium lattice constant of fcc-Fe different data are found in literature $\left[a_{F e}^{f c c}=3.6468 \AA\right.$ (Ref. 38) and $a_{F e}^{f c c}$

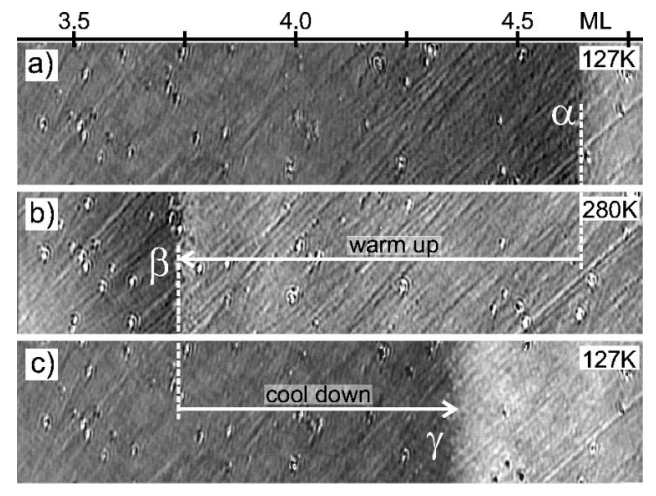

FIG. 4. Thermally induced shift of the reorientation transition. (a) - (c) $t_{\text {crit }}$ changes from $\alpha$ to $\beta$ when raising the temperature from $127 \mathrm{~K}$ to $280 \mathrm{~K}$. Cooling back down to $127 \mathrm{~K}$ shifts the SRT to $\gamma \neq \alpha$.

$=3.59 \AA$ (Ref. 23)], leading to in-plane strain of opposite $\operatorname{sign}\left[\varepsilon_{\|}=-0.88 \%\right.$ vs $\varepsilon_{\|}=+1.54 \%, a_{C u}=3.6147 \AA$ (Ref. 38)]. The observation of tensile stress in the Fe film during the growth on $\mathrm{Cu}(001)$ (Refs. 25 and 39), supports an expanded lattice constant in the pseudomorphic Fe layer; thus an $\varepsilon_{\|}=+1.54 \%$ is assumed for the calculation. The perpendicular strain follows from the average interplanar spacing of $d=1.87 \AA$ (Ref. 24) to $\varepsilon_{\perp}=+5 \%$. The magnetoelastic coupling $B_{1}$ was found to be strain dependent, ${ }^{25}$ giving a $B_{1}^{\text {eff }}$ $=13.5 \mathrm{MJ} / \mathrm{m}^{3}$ for the pseudomorphic $4 \mathrm{ML}$ Fe. This value differs significantly in magnitude and sign from the respective bulk value, which was used in all previous calculations. Using $M_{S}=1751 \mathrm{kA} / \mathrm{m}, K_{f-v a c}=0.89 \mathrm{~mJ} / \mathrm{m}^{2}$ (Ref. 26), and $K_{f-s}=0.6 \mathrm{~mJ} / \mathrm{m}^{2}$ (Ref. 27) gives the thickness dependence of $\Delta F_{t o t}$ as depicted in Fig. 3(c) as a solid line. To reproduce the shift of $t_{c r i t}$ due to $\mathrm{CO}$ in Fig. 3(b), a changed $K_{f-v a c}$ $=0.63 \mathrm{~mJ} / \mathrm{m}^{2}$ has to be assumed, giving the thickness dependence of $F_{\text {tot }}$ shown as a dashed line in Fig. 3(c). The $K_{f-s}$ remains unchanged. The oxygen-induced reorientation can only be explained by a change in sign of $K_{f-v a c}$ with $K_{f-v a c}+K_{f-s}>0$. It is worth noting that despite the large $B_{1}^{e f f}$ the magnetoelastic contribution is not sufficient to compete with the surface anisotropy and thus to alter the easy axis.

\section{B. Thermally induced reorientation transition}

Thermal treatment of the film can change the spin reorientation transition reversibly ${ }^{40}$ as well as irreversibly. Reversible changes might arise from a pure temperature dependence of the anisotropy constants and will vanish after subsequent cooling of the film back to the starting temperature. In contrast to that, thermally activated irreversible changes due to diffusion or changes of the film structure and morphology, etc., will remain. We performed a simple experiment on a LT-grown Fe wedge to separate temperaturedependent reversible changes and irreversible changes in $t_{\text {crit }}$ due to annealing using the Kerr microscope. The remanent polar magnetization of an $\mathrm{Fe}$ wedge after a magnetic field was applied is shown in Fig. 4(a). The change in brightness at the crossover from perpendicular magnetization (dark area) to in-plane magnetization (bright area) marks the criti- 
cal thickness $\alpha=4.65 \mathrm{ML}$ of this wedge at the temperature of $127 \mathrm{~K}$, after being warmed up to $270 \mathrm{~K}$. Further annealing of this film to $T_{a}=280 \mathrm{~K}$ shifts the critical thickness towards smaller Fe thickness $\beta=3.73$ ML in Fig. 4(b). Subsequent cooling of the sample back to $127 \mathrm{~K}$, followed by remagnetization in $H_{\perp}=25 \mathrm{mT}$, brings $t_{\text {crit }}$ back to a higher film thickness $\gamma=4.35 \mathrm{ML}$, but the critical thickness as in Fig. 4(a) cannot be attained. Instead, any further change of $T$ between 127 and $280 \mathrm{~K}$ reversibly switches $t_{\text {crit }}$ between $\beta$ and $\gamma$.

The dependence of the SRT on the annealing temperature in the temperature range between $100 \mathrm{~K}$ and RT is currently being studied in more detail and will be published elsewhere. The data obtained by Kerr microscopy are compared with information about the film structure as obtained by variabletemperature STM (VT-STM). Preliminary results show a strong nonlinear dependence of $t_{c r i t}$ on the annealing temperature, accompanied by temperature-driven changes of the film morphology.

We argue that the difference between $t_{c r i t}$ in Figs. 4(a) and 4(c), both images obtained at $T=127 \mathrm{~K}$, reflects irreversible changes of the film structure due to the annealing. On the other hand, the reversible shift in $t_{c r i t}$ from $\beta$ to $\gamma$ after cooling back from $280 \mathrm{~K}$ seems to be purely due to a temperature dependence of the anisotropy constants, i.e., $K(T)$. In this picture, thermally activated changes in the film structure have happened during warming up the film and remain unchanged when cooling back to $127 \mathrm{~K}$. Thus, the experiment shown allows us to separate the magnetic reorientation due to temperature-driven structural changes from the purely reversible effect due to temperature-dependent anisotropy constants.

To fully understand the observed effect more data about the film structure as a function of temperature are needed. Assuming that annealing the film reduces the film roughness, such a smoothing would increase the surface anisotropy shifting the $T_{\text {crit }}$ towards higher thickness. ${ }^{41}$ The observed decrease of $t_{c r i t}$ for $T_{a}=280 \mathrm{~K}$ thus cannot be explained by a surface smoothing. The details of the film structure after annealing are currently being investigated with VT-STM. One might speculate whether the bcc crystallites found in STM studies ${ }^{42}$ grow during annealing, making the film more bcc like and causing a switch of the easy axis into the film plane. This idea might be supported by recent results showing drastic changes of the morphology of a RT-grown fcc Fe film on $\mathrm{Cu}(001)$ after cooling and subsequent warming up to $300 \mathrm{~K}$, ascribed to a transition of the film to bcc structure. ${ }^{9}$ Cycling the temperature was indeed found to increase the bcc needle size noticeably in Fe films grown at $300 \mathrm{~K} .{ }^{43}$ The film structure therefore seems to depend sensitively on the film temperature in the temperature range below RT. On the other hand, one also has to take into account temperatureactivated diffusion between the film and substrate, as this is known to alter the magnetic anisotropy energy. ${ }^{8}$ It is interesting to note that other authors ${ }^{13}$ find a reorientation from in-plane to perpendicular for LT-grown $6 \mathrm{ML} \mathrm{Fe} / \mathrm{Cu}(001)$ after annealing above $T_{a}>350 \mathrm{~K}$, accompanied by a surface smoothing. Although such high temperatures were not investigated in this work, it contradicts the shift of $t_{c r i t}$ towards smaller film thickness as observed in Fig. 4(b), as well as results from RT-annealed films in Ref. 40 and images in Ref. 9.

\section{CONCLUSION}

The susceptibility of the spin reorientation transition in LT-grown $\mathrm{Fe} / \mathrm{Cu}(001)$ to adsorbate coverage and thermal treatment underlines the delicate balance between electronic and structural properties on the one hand and magnetic properties on the other hand in this system. Kerr Microscopy experiments with adsorbates $\mathrm{CO}$ and $\mathrm{O}_{2}$ have been performed. A strong dependence of the magnetic anisotropy of the film on oxygen and $\mathrm{CO}$ coverage was found. The data presented in this paper underline the dominant role of the film-vacuum interface. This supports the usual picture of a balance between the surface and volume anisotropy as the determining factor for the orientation of the easy axis of magnetization. The spin reorientation can be modeled by a modified surface anisotropy constant $K_{f-v a c}$. The thermally induced changes of the reorientation transition are partly reversible and partly irreversible. This is indicative of temperature-dependent anisotropy constants, but also suggests structural changes of the film due to annealing. The latter points out the sensitive relationship between the film structure and its magnetism and is subject to further investigation.
*Electronic address: a.enders@fkf.mpg.de; URL: http://www.mpistuttgart.mpg.de/kern

${ }^{1}$ R. Allenspach, J. Magn. Magn. Mater. 129, 160 (1994).

${ }^{2}$ A. Kirilyuk, J. Giergiel, J. Shen, M. Straub, and J. Kirschner, Phys. Rev. B 54, 1050 (1996).

${ }^{3}$ R. Vollmer and J. Kirschner, Phys. Rev. B 61, 4146 (2000).

${ }^{4}$ A. Bauer, Habilitation, Freie Universität Berlin, 2000.

${ }^{5}$ A. Biedermann, M. Schmid, and P. Varga, Phys. Rev. Lett. 86, 464 (2001).

${ }^{6}$ D. Li, M. Freitag, J. Pearson, Z. Qiu, and S. Bader, Phys. Rev. Lett. 72, 3112 (1994).

${ }^{7}$ V. Morruzi, P. Marcus, K. Schwarz, and P. Mohn, Phys. Rev. B 34, 1784 (1986).
${ }^{8}$ B. Újfalussy, L. Szunyogh, and P. Weinberger, Phys. Rev. B 54, 9883 (1996).

${ }^{9}$ D. Qian, X.F. Jin, J. Barthel, M. Klaua, and J. Kirschner, Phys. Rev. Lett. 87, 227204 (2001).

${ }^{10}$ K. Man, W. Ling, S. Paik, H. Poppa, M. Altman, and Z.Q. Qiu, Phys. Rev. B 65, 024409 (2001).

${ }^{11}$ R. Allenspach and A. Bishof, Phys. Rev. Lett. 69, 3385 (1992).

${ }^{12}$ J. Giergiel, J. Shen, J. Woltersdorf, A. Kirilyuk, and J. Kirschner, Phys. Rev. B 52, 8528 (1995).

${ }^{13}$ E. Mentz, D. Weiss, J. Ortega, A. Bauer, and G. Kaindl, J. Appl. Phys. 82, 482 (1997).

${ }^{14}$ J. Bansmann, M. Getzlaff, C. Westphal, and G. Schönhense, J. Magn. Magn. Mater. 117, 38 (1992). 
${ }^{15}$ M. Salvietti, P. Ferro, R. Moroni, M. Canepa, and L. Mattera, Surf. Sci. 377-379, 481 (1997).

${ }^{16}$ J. Shen, A.K. Swan, and J.F. Wendelken, Appl. Phys. Lett. 75, 2987 (1999).

${ }^{17} \mathrm{R}$. Vollmer (private communication).

${ }^{18}$ R. Vollmer, S. van Dijken, M. Schleeberger, and J. Kirschner, Phys. Rev. B 61, 1303 (2000).

${ }^{19}$ M.A. Torija, J.P. Pierce, and J. Shen, Phys. Rev. B 63, 092404 (2001)

${ }^{20}$ R. Vollmer, T. Gutjahr-Löser, J. Kirschner, S. van Dijken, and B. Poelsema, Phys. Rev. B 60, 6277 (1999).

${ }^{21}$ W. Ling and Z. Qiu, Phys. Rev. B 63, 024408 (2000).

${ }^{22}$ D. Spišak and J. Hafner, Phys. Rev. B 64, 094418 (2001).

${ }^{23}$ F. Jona and P.M. Marcus, Crit. Rev. Surf. Chem. 4, 189 (1994).

${ }^{24}$ S. Müller, P. Bayer, A. Kinne, C. Reischl, R. Metzler, and K. Heinz, Surf. Sci. 331-333, 723 (1995).

${ }^{25}$ T. Gutjahr-Löser, Ph.D. thesis, Martin-Luther-Univ. Halle Wittenberg, 1999.

${ }^{26}$ B. Heinrich, Z. Celinski, J.F. Cochran, A.S. Arrott, and K. Myrtle, J. Appl. Phys. 70, 5769 (1991).

${ }^{27}$ U. Gradmann, in Handbook of Magnetic Materials, edited by K. H. J. Buschow (Elsevier Science Publishers B. V., 1993), Vol. 7.

${ }^{28}$ D. Peterka, G. Haas, A. Enders, and K. Kern, (unpublished).

${ }^{29}$ J. Thomassen, B. Feldmann, and M. Wuttig, Surf. Sci. 264, 406 (1992).
${ }^{30}$ J. Giergiel, J. Kirschner, J. Landgraf, J. Shen, and J. Woltersdorf, Surf. Sci. 310, 1 (1994).

${ }^{31}$ A. Vaterlaus, C. Stamm, U. Maier, M.G. Pini, P. Politi, and D. Pescia, Phys. Rev. Lett. 84, 2247 (2000).

${ }^{32}$ H. Zillgen, B. Feldmann, and M. Wuttig, Surf. Sci. 321, 32 (1994).

${ }^{33}$ B. Ujfalussy and P. Weinberger (private communication).

${ }^{34}$ J. Radnik, E. Chopovskaya, M. Grüne, and K. Wandelt, Surf. Sci. 352-354, 268 (1996).

${ }^{35}$ G. Blyholder, J. Phys. Chem. 68, 2772 (1964).

${ }^{36}$ P. Ferro, R. Moroni, M. Salvietti, M. Canepa, and L. Mattera, Surf. Sci. 407, 212 (1998).

${ }^{37}$ R. Moroni, F. Bisio, M. Canepa, and L. Mattera, Appl. Surf. Sci. 175-176, 797 (2001).

${ }^{38}$ Eckerlin/Kandler, in Landolt-Börnstein, New Series, Group III, Vol. 6, (Springer, Berlin, 1971), p. 9.

${ }^{39}$ T. Gutjahr-Löser, D. Sander, and J. Kirschner, J. Appl. Phys. 87, 5920 (2000).

${ }^{40}$ D.P. Pappas, K.-P. Kämper, B.P. Miller, H. Hopster, D.E. Fowler, A.C. Luntz, C.R. Brundle, and Z.-X. Shen, J. Appl. Phys. 69, 5209 (1991).

${ }^{41}$ P. Bruno, J. Phys. F: Met. Phys. 18, 1291 (1988).

${ }^{42}$ A. Biedermann, R. Tscheliessnig, M. Schmid, and P. Varga, Phys. Rev. Lett. 87, 086103 (2001).

${ }^{43}$ A. Biedermann (private communication). 